\title{
A correlational analysis of human cognitive activity using Infrared Thermography of the supraorbital region, frontal EEG and self-report of core affective state.
}

\author{
by S.D.Jenkins* and R.D.H.Brown*
}

\author{
*UWTSD Swansea, Mount Pleasant, Swansea, South Wales, UK SA16ED, sean.jenkins@sm.uwtsd.ac.uk
}

\begin{abstract}
Using Infrared Thermography, three-way statistical correlation between forehead temperatures, frontal EEG and self-reports of core affect was applied in an experimental study to establish the underlying causal relationships between cognitive activity and supraorbital blood flow. Sub-groups were identified within the sample using the differentia of tonic forehead temperature asymmetry. Comparison between these groups showed that the so-called Right Dominant temperature sample exhibited statistically significant correlations between asymmetry in frontal alpha, forehead temperature and self-reported arousal throughout the experimental interactions. Individuals exhibiting this trait demonstrated greater reactivity to variation in the cognitive challenge of the tasks, presenting more readily measurable and distinctive patterns of asymmetric frontal cortical activity and temperature change. Detailed analysis showed significant patterns of correlation between hemispherical changes in specific beta frequencies, forehead temperature and self reported arousal and task engagement, indicating that forehead temperature dynamics reflected the underlying variation in cognitive demand evoked by the experimental stimuli.
\end{abstract}

\section{Introduction}

Recent empirical studies using Infrared Thermography (IRT) indicate that changes in blood flow in the forehead are related to changes in cognitive and affective processes. Pavlidis et al have been pre-eminent in the publication of developments in the application of IRT in cognitive workload assessment since their initial report, in 2000 [1], on increases in temperature of the periorbital region arising from 'fight or flight' autonomic response to elevated stress. However, since 2005 this group has shifted its attention to the supraorbital region. The research group's diversification of interest to the supraorbital vessels is based on the premise that temperature increases are correlated with metabolic increases in this region of interest. This is presumed to be due to the influence of muscle activation of the forehead muscle group, specifically the corrugator muscle used in frowning, that Puri et al [2] described as a manifestation of prolonged stress arising from mental effort. This proposition has been carried through in the group's more recent studies of cognitive workload assessment using this region of interest to monitor and control difficulty levels of a video game [3], analyse cognitive loading due to multi-tasking during a driving simulation [4], assess mental challenge [5] and in subsequent deceit detection studies [6,7]. Although it is feasible that the action of the corrugator musculature may contribute to the observed phenomena no empirical evidence has been provided to support this, for example by correlating temperature measures with facial Electromyogram (EMG). While it is noted that there are conflicting reports in the literature which suggest that the nasal region is a more reliable indicator of cognitive loading [8,9], physical arousal [10], positive affective behaviour [11] or mental arousal [12], other researchers have also reported that forehead temperature dynamics are indicative of changes in mental load arising from cognitive stressors [13,14], and that affective classification systems based on temperature measures of this region can discriminate between experienced levels of positive and negative valence [15], supporting the proposition that changes in blood flow in the supraorbital vessels correlate with changes in cognitive loading and affective processing. However, the underlying causation of this observed phenomenon has yet to be effectively established.

Although theories relating facial blood flow (FBF) and cerebral blood flow (CBF) such as Zajonc's Vascular Theory of Emotional Efference [16,17] have been proposed, very few detailed experimental studies have been undertaken to test them using IRT. This is all the more remarkable considering the direct relationship observed between the target blood vessels of the forehead region and those branches of the vascular system also implicated in the supply of blood to the frontal lobes of the brain - the supraorbital and supratrochlear arteries of the forehead are supplied by the internal carotid artery, via the ophthalmic artery that branches from the frontopolar branch of the anterior cerebral artery, a key source of blood supply to the frontal lobes of the brain. Furthermore, the literature on theories relating to the lateralisation of brain function [18], asymmetrical control of the autonomic nervous system (ANS) $[19,20]$ and the role of somatosensory and homeostatic processes in affective processing and cognition $[21,22,23]$ suggest a line of research that has yet to be effectively explored in studies using IRT.

In contrast with IRT studies, extensive investigations using the frontal Electroencephalogram (EEG) have established important patterns of cortical activity and hemispheric involvement in sensory perception and cognitive performance [24]. Frontal EEG asymmetry has also been related to affective information processing, expression and response. Measurement of asymmetrical alpha activity of the frontal EEG has been the main paradigm in the exploration of responses to a variety of affective and cognitive stimuli and there is an extensive body of literature in this field exploring the importance of resting activity (tonic) and state activation (phasic) changes in frontal EEG asymmetry $[25,26]$. One of the main proposals arising from this body of work is that electrophysiological markers of left and right 
brain activation are suggested to be an index of motivational/affective traits and states [27]. By comparison, there is little research using IRT to specifically explore hemispherical temperature differentiation of the supraorbital region of interest, $\mathrm{ROI}$, and the potential relationship between temperature dynamics of this site and underlying cortical activity. While temperature asymmetry at other sites of the face has been considered [28,29], to date just a handful of studies have directly addressed this gap in knowledge. The anatomical relationship between the supraorbital vessels and those supplying the frontal lobes of the brain suggests a direct comparison is needed between the electrophysiological markers of frontal cortical activity and forehead temperature dynamics. Only two documented studies, outside the work of the authors, have attempted to correlate facial skin temperature directly with cortical activation. Genno et al. [31] compared EEG alpha/beta power ratio at the centreline of the parietal region with nose temperature, measured by thermistor not IRT, and electrocardiograms to measure fatigue during a repetitive task. Nozawa and Tacano [32] compared alpha attenuation at the occipital lobe with temperature change at the nose and self-reports of arousal. Neither of these studies used the frontal alpha asymmetry metric, which is more commonly applied in psychophysiology studies, nor have they considered the supraorbital ROI. By contrast, previous work of the authors has shown that mentally challenging activities give rise to changes in human forehead temperature that are correlated with changes in total frontal lobe EEG activity [33]. It was hypothesised that variation in the underlying cognitive activity was influential in the observed changes in blood flow in the supraorbital region. Relationships between temperature, total EEG activity and the subjective feelings of participants were observed in this experiment that supported this hypothesis. Critically, these relationships appeared to be pronounced when the sample was disaggregated on the basis of tonic forehead temperature asymmetry.

This paper reports on a detailed extension of the previous work of the authors. First, two levels of interaction, non-contact and contact, with a computer-based stimulus were used to establish if temperature dynamics of the supraorbital region of the forehead are related to variation in specific cognitive processes elicited by the different interactive stimuli. Secondly, the differentia of tonic forehead temperature asymmetry was applied to the analysis to explore the relationship with frontal alpha asymmetry and the influence of this trait on psychophysiological responses to the stimuli. By simultaneously measuring temperature using IRT and cortical activity using specific frequency bands of the EEG during the course of well-defined tests, this comparison has been achieved and the results presented here. A third variable, known as 'core affect' [34,35], was measured by means of a questionnaire, in order to assess any potential relationships between subjective feelings and the objective physiological measures obtained by IRT and EEG.

\section{Methodology}

Three-way correlation between stimulus induced changes in Temperature, EEG frequency and Subjective Affect, so-called 'methodological triangulation', was applied in an experimental study undertaken with an all male sample $(N=33)$ of undergraduate design students using a repeated measures, within subjects design. An all male sample was chosen in order to avoid potential variability that mixed gender sample could induce, as previously reported $[36,37,38]$. Two interactive stimuli, so-called MAP and iMAP, were devised to simulate different levels of cognitive demand requiring non-contact and contact based interaction. The stimuli developed here were based on an established test of executive function, known as the 'Zoo Map Test', which is part of a battery of psychological tests known as BADS - Behavioural Assessment of Dysexecutive Syndrome [39]. This test was specifically selected because it has been shown to have good ecological validity in that the ability to perform well on the task was found to be correlated with not only subjective reporting of executive symptoms [40] but also a further test of executive function that is conducted in a real-life setting [41]. In this particular test, subjects are required to formulate a route around a map of a fictitious zoo, without contravening a set of rules. To increase the relevance of the test, a common everyday activity - making a hot drink was selected to replace the zoo scenario to ensure there would be a high degree of familiarity for all test subjects and to incorporate the simulation of an everyday interaction scenario as many of the previously reviewed studies used stimuli designed to induced high levels of anxiety, stress or cognitive load [e.g. 1,2,5,10,36]. An issue with the repeated measures experimental design is the potential threat to internal validity arising from confounding variables such as order or transfer effects $[42,43]$. To reduce such effects presentation of the stimuli was alternated across the participants using a Latin Square design. The experimental procedure consisted of six stages; (1) Preparation and acclimatisation of the participant, (2) pre-test affective self report, (3) measurement of response to test stimulus A (MAP) using IRT and EEG, (4) post-test affective self report, (5) measurement of response to test stimulus B (iMAP) using IRT and EEG, (6) posttest affective self report. The test order was alternated; half the group were presented with the stimuli in the order $A \mid B$ and half presented with the stimuli in the order $\mathrm{B} \mid \mathrm{A}$. Due cognisance was given to the established protocols for effective and reliable thermal imaging of human participants $[44,45,46]$ in the preparation, acclimatisation and recording of participants.

The experiment took place over eights separate days; ambient temperatures of between $18.6^{\circ} \mathrm{C}$ and $20.9^{\circ} \mathrm{C}$ were recorded at these sessions with temperature stability within each session better than $\pm 0.5^{\circ} \mathrm{C}$. Temperature measurements were obtained using a Cedip Infrared Systems (acquired by FLIR Systems Inc. in 2008) Titanium 560M internally cooled camera with a $25 \mathrm{~mm}$ (FOV $21^{\circ}$ ) optical lens. The camera has a $640 \times 512$ pixel / 14 bit detector focal plane array and is capable of capturing images at a maximum full frame rate of up to $380 \mathrm{~Hz}$. The camera's Indium Antimonide $(\mathrm{InSb})$ sensor is highly sensitive to the mid wave infrared spectral band $(3.6-5.1 \mu \mathrm{m})$ with a specified thermal sensitivity of $<25 \mathrm{mK}$ or $0.025^{\circ} \mathrm{C}$ (Noise Equivalent Temperature Differential) at $25^{\circ} \mathrm{C}$ and spatial resolution $1.1 \mathrm{mRAD}$. This corresponds to a potential variation in measurements of $\pm 0.1 \%$ at $25^{\circ} \mathrm{C}$. The sensitivity and stability of the camera 
was tested by comparison with a stable black body calibration source (Omega BB702) at a temperature corresponding approximately to the mid level in the temperature span of the forehead temperatures measured in the experiment. The variability measured was $0.06 \%$ of the mean temperature, $36.29^{\circ} \mathrm{C}$. This variation is within the stated specification of the camera. The stability of system was also monitored during the course of the experiments by measuring the temperature of a stable target region in the background of the recorded image sequences. The temperature fluctuations measured were, typically, $\pm 0.04 \%$ of the mean temperature, $21.52^{\circ} \mathrm{C}$. These measurements confirm that the camera was stable throughout the experimental recordings and that the stability of the infrared camera was within $\pm 0.1 \%$ of the mean value of the background target reference temperature.

The camera was connected to a DELL laptop via a Camlink interface for digital video capture. All radiometry parameters were programmed directly into the software for correction prior to recording. The video sequences were directly acquired using Cedip's Altair v5.8 software. The video standard used was PAL $(50 \mathrm{~Hz})$. The sampling frame rate selected for video capture was $50 \mathrm{~Hz}$; sub-sampling at a rate of 1:25 frames (two frames per second) at a size of $320 \times 256$ pixels. Random motion compensation was undertaken on all captured video sequences using a post-processing module. Reference marks were applied to participants' foreheads to provide positional targets for tracking. A flexible plastic template with a grid of holes at $5 \mathrm{~mm}$ intervals was used to 'mark-up' the subject's forehead using a metallic gel ink pen. The metallic particles in the ink possess lower emissivity properties to the known emissivity of human skin, therefore ensuring that the reference marks would be visible in the thermal images. Eight reference points were marked to provide targets for post-process motion compensation and ensure accurate and consistent positioning of measurement tools defining regions of interest in subsequent thermal image analysis. To accommodate the anthropometric variation in forehead dimensions the same method was used for each subject: the centreline of the forehead was defined with two points, one positioned just above the nasion - a recessed point between the forehead and nose - to a point below the hairline. The outer edges of the eyes were used to define a further set of three vertical reference points on each side of the forehead indicating the pronounced transition in surface plane direction of the forehead (frontal bone) along the temporal line. This demarcation ensured that the area tools were placed on anterior surfaces only during analysis. Each original video sequence was loaded in to the motion compensation module. Vectors were created between the reference targets as illustrated on the left in Figure 1 and automatic tracking was applied. On completion of this process, motion compensation was calculated and the new sequence was rendered and saved for analysis. A standard circle tool with an area of 610 pixels was used to measure the temperature of the right and left supraorbital regions, shown on the right in Figure 1. The mean temperatures of the right and left ROl's were used to produce timing graphs of temperature change over the 360 -second (720 frames) sequence. The temperature range in the images was 'squeezed' to a $4^{\circ} \mathrm{C}$ span and histogram equalisation was applied.

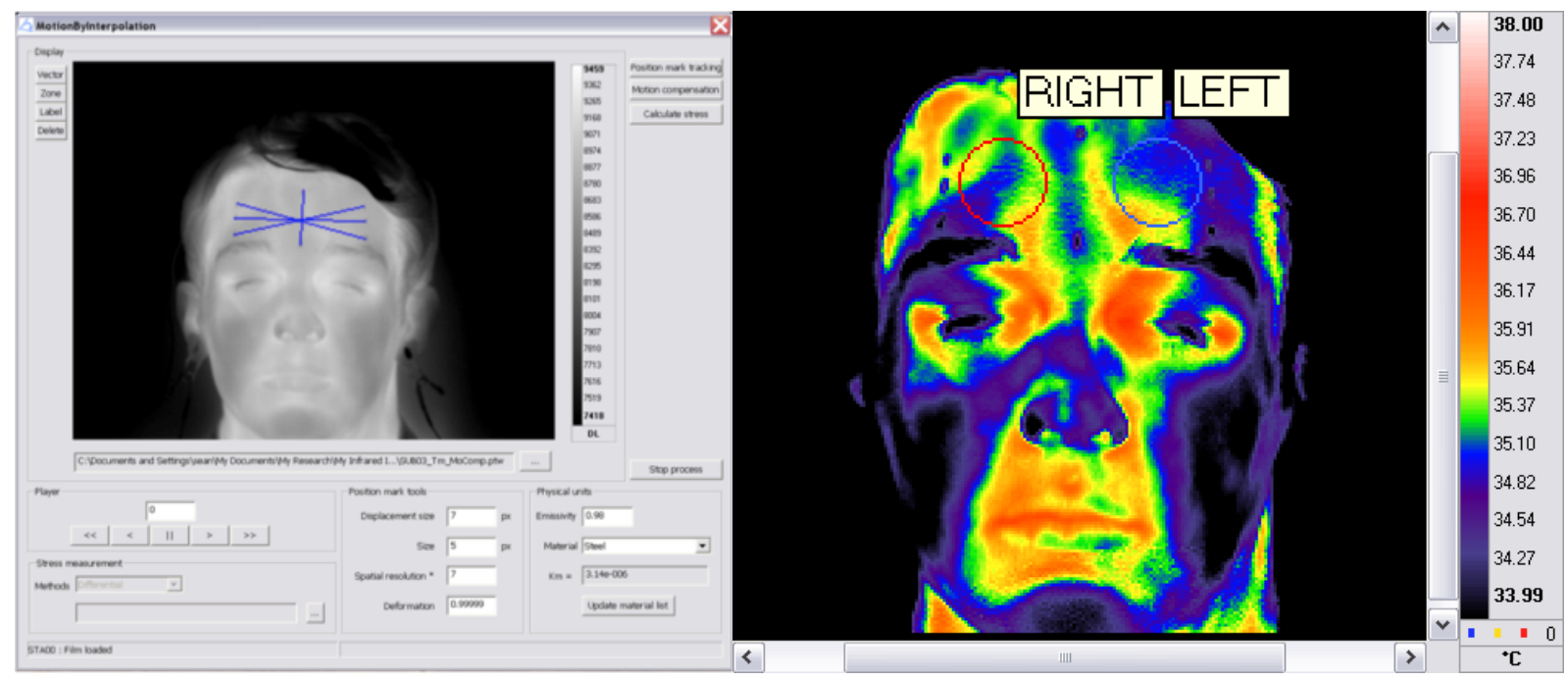

Fig 1. Motion compensation applied to ensure accuracy of temperature measurements at Supraorbital ROI's.

Frontal EEG measurements were obtained using a MindMedia Nexus10. EEG data was recorded from specific locations according to the International 10-20 electrode placement system [24,47,48]. A reference recording montage was used. The locations for the scalp electrodes were F3 (Frontal Lobe: Left Hemisphere) and F4 (Frontal Lobe: Right Hemisphere), the reference electrodes were placed behind the ear on each mastoid bone M1 and M2. The ground electrode was placed at CZ. The NeXus-10 is a battery powered 10 -channel physiological monitoring and biofeedback platform that utilises Bluetooth 1.1 class 2 wireless communications to facilitate wireless transfer of data from the recorder to the monitoring computer. The hardware offers data acquisition rates of up to 2048 samples per second. BioTrace+ v2009 software was used in the recording and analysis of the EEG. BioTrace+ provides ten 'real' data channels and a further 70 'virtual' computed data channels. The first two channels are for the EEG sensor inputs (Input $A$ \& $B: D C-800 \mathrm{~Hz}$ ) that are sampled at a rate of 256 samples per second. The EEG raw signal is derived from the 
sensor input that is passed through a $3^{\text {rd }}$ order band pass filter between specified frequencies. The system records the total EEG over a frequency range of $1-64 \mathrm{~Hz}$. The separate constituent frequency bands considered in this experiment were derived in the same manner. For example, the alpha amplitude was computed from the alpha wave, filtered from the raw EEG signal in the range $8-12 \mathrm{~Hz}$. The amplitude is derived from the wave by computing an RMS value 32 times per second over an epoch size of $1 / 8^{\text {th }}$ of a second. It is almost inevitable that some artefact (biological or non-biological) will contaminate the raw EEG signal; due cognisance was given to protocols for eliminating the deleterious effects of artefact in the EEG $[48,50]$. BioTrace+ offers an automatic artefact rejection function for EMG artefact and $50 \mathrm{~Hz}$ interference and screen-based instruments for monitoring the integrity of electrode contact.

Although the specific frequency ranges may vary in the EEG and biofeedback literature, it is established that the general bandwidths of alpha and beta correspond with particular types of mental state. The constituent frequencies of the total EEG analysed in this experiment were alpha $(\alpha)$ at $8-12 \mathrm{~Hz}$ and beta $(\beta) 12-23 \mathrm{~Hz}$. The beta frequency band was sub-divided into sensory-motor rhythm or SMR $(s \beta)$ at $13-15 \mathrm{~Hz}$, low beta $(I \beta)$ at $16-20 \mathrm{~Hz}$ and high beta $(h \beta)$ at $20-23 \mathrm{~Hz}$.

Greater alpha activity between $8-12 \mathrm{~Hz}$ is normally observed when subjects are in a relaxed, resting state (eyes closed or open); with the onset of mental activity alpha waves generally reduce in amplitude [24]. Anxiety, stress and mental loading result in decreased alpha activity in normal subjects [49]. Sudden alertness or concentration on mental tasks results in a phenomenon known as alpha blocking or alpha desynchronisation. By subtracting the left hemisphere value from the right hemisphere value an index of alpha asymmetry is obtained; because alpha activity is considered to be inversely related to brain activation [50], positive values describe higher right hemisphere alpha which indicates greater left hemisphere activation, whereas negative values describe higher left hemisphere alpha indicating greater right hemisphere activation.

Beta rhythms typically replace alpha during diffuse arousal and cognitive activity and are normally associated with an externally focused, attentive and problem-solving state. Beta can be sub-divided into narrower frequency ranges that correspond to precise kinds of cortical functioning. Activity in the range of $13-15 \mathrm{~Hz}$ is labelled sensorimotor rhythm when found across the sensor-motor strip of the cortex towards the posterior of the frontal lobe. It has been associated with reduced sensory input and motor output and appears to be indicative of a calm attentive mental state with increased 'reflecting before acting'. Activity in the range of $16-20 \mathrm{~Hz}$ is labelled low beta and is associated with problem solving. Activity in the range of $20-23 \mathrm{~Hz}$ is labelled high beta and is observed more often in anxious subjects and may correlate with emotional intensity. An increase in high beta can be correlated with (1) productive cognitive work, (2) productive but too intense cognitive work or (3) unproductive intense cognitive work or anxious thinking [49].

Self-reports of core affect were obtained using single item semantic differential scales, which have been found to have good validity and excellent reliability when compared to multi-item questionnaires [51] and have been used in several of the studies reviewed earlier $[32,37]$. Each item comprised of a bi-polar pair of adjectives presented in the form of visual analogue scales. The dimensions of core affect were recorded by the participants on $100 \mathrm{~mm}$ scales between INACTIVE (Drowsy) and ACTIVATED (Alert) for arousal, and between UNPLEASANT and PLEASANT for valence. A third item reflecting task engagement was measured on a scale between DISAGREEABLE and AGREEABLE.

Measures of state change in temperature $(T)$, EEG $(E)$ and core affect $\left(A_{r}\right.$ and $\left.V_{a}\right)$ were derived from the raw data by subtracting the baseline measure from the test measure for each variable. Change values in IRT and EEG measures were calculated for both the left and right hemispheres. Measures of asymmetry in temperature (T) and EEG (E) were derived from the raw data by subtracting the left hemisphere measure from the right hemisphere measure for each variable.

Non-parametric statistical tests were used in the analysis, as these do not assume that the data is normally distributed. Spearman's (rho) Rank Order Correlation Coefficient $(\rho)$ was selected to measure the strength of the relationships between the variables; regression analysis was used to establish how well a relationship between the variables fits a linear model. Where comparisons were required between paired data the Wilcoxon Signed Ranks Test was used to establish significant differences. Where comparisons were required between the two separate groups within the sample the Mann-Whitney U Test was selected.

\section{Results}

As in previous work published by the authors, it was observed that the sample population was complex. Subgroups were identified within the sample using the differentia of tonic forehead temperature asymmetry. It was observed that there were twenty participants in the sample who exhibited higher left side forehead temperatures at the start of the recording period compared to thirteen who demonstrated higher right side forehead temperatures. The right hemisphere temperatures were comparable between the groups and there was no significant difference. Left hemisphere temperatures were significantly different between the groups. The Left Dominant group exhibited a higher mean left hemisphere initial baseline forehead temperature of $33.64^{\circ} \mathrm{C}(\mathrm{SD}=.49)$ in comparison to the Right Dominant groups left hemisphere temperature of $33.29^{\circ} \mathrm{C}(S D=.39)$. The difference between these mean values of $0.35^{\circ} \mathrm{C}$ was statistically significant at the $95 \%$ level $(p=.025)$. Visual analysis of thermal timing graphs showed that the trait temperature 
asymmetry of each group remained consistent throughout the experimental procedure, as observed in the previous experiment. Statistical analysis confirmed that the differences between left and right hemisphere temperatures of each group remained significant throughout the experimental procedure (Initial Baseline, MAP, iMAP) at the 99\% level $(p \leq .002)$.

Hemispherical differences have been related to cognitive performance and affective traits/states and measurement of asymmetrical activity in the alpha frequency of the frontal EEG has been the main paradigm used in the exploration of these processes. Direct comparison using this asymmetry metric showed that so-called Right Dominant cases, possessing lower left hemisphere forehead temperatures, exhibited statistically significant correlations between asymmetry in frontal alpha, forehead temperature and self-reported arousal. The scatter plot in Figure 2 below depicts the significant positive association observed for this sub-group (indicated by the $\Delta$ symbol) between initial baseline frontal alpha asymmetry and forehead temperature asymmetry $\left(\mathrm{T}_{\mathrm{A}}: \alpha_{\mathrm{A}}: \rho=.577, N=13, p \leq 0.05\right)$. Regression analysis confirms in this case that there is a strong linear relationship between the variables and that the gradient of the curve is statistically significant at the $95 \%$ level $(t=2.914, p=.014)$. This relationship was observed only in the Right Dominant sub-group, both during baseline measurements and during the experimental interactions with the stimuli. A significant negative correlation was observed between baseline temperature asymmetry and self-reported arousal $\left(T_{A}: A_{r}: \rho=-.637, N=13\right.$, $\mathrm{p} \leq 0.05)$. Regression analysis indicates that the significance of the linear relationship is just below the $95 \%$ level $(t=2.140, p=.056)$. Similarly, a negative association was also observed between baseline alpha asymmetry and selfreported of arousal $\left(\alpha_{A}: A_{r}: p=-.520, N=13, p \leq 0.1\right)$. Although the significance of the correlation coefficient falls just below the $95 \%$ level regression analysis indicates that there is a strong linear relationship between the variables and the gradient of the curve is statistically significant at the 0.05 level $(t=2.301, p=.042)$.

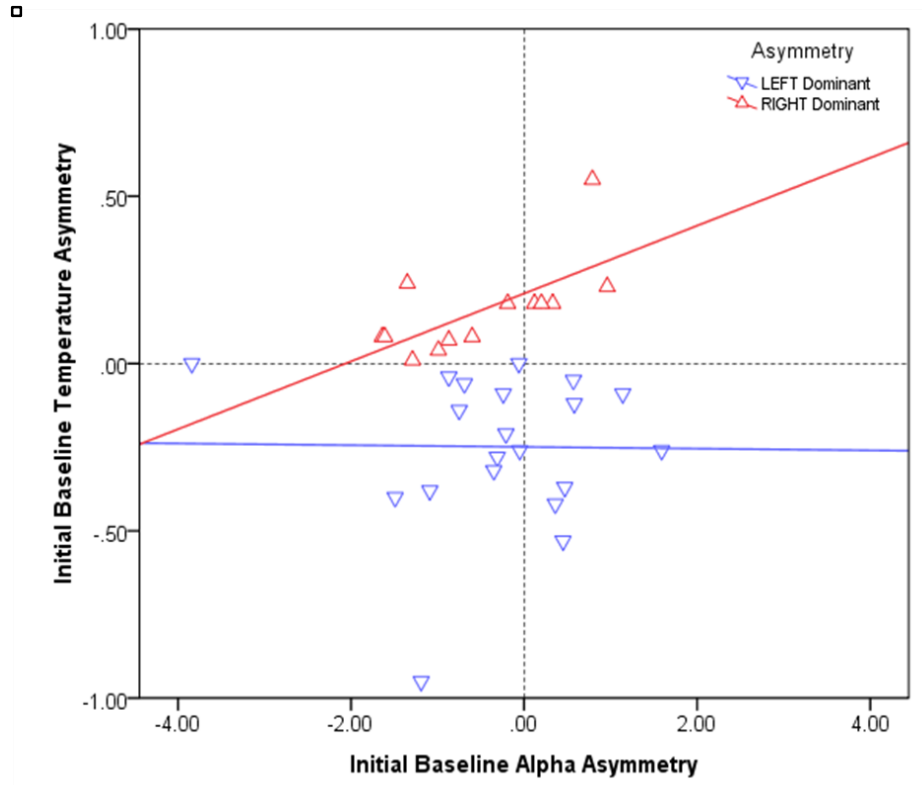

Fig 2. Tonic trait relationship between $T_{A}: \alpha_{A}$ for LEFT and RIGHT dominant sub-groups.

The Right Dominant group exhibited a higher baseline level of self-reported arousal than the Left Dominant group, a difference that was statistically significant at the $95 \%$ level $(p=0.43)$. The results suggest that the observed temperature asymmetry trait is indicative of greater relative dominance of sympathetic function in autonomic nervous system balance and a higher tonic state of psychophysiological arousal. Individuals exhibiting this tonic trait demonstrated greater reactivity to variation in the cognitive challenge of the tasks, presenting more readily measurable and distinctive patterns of asymmetric cortical activity and temperature change. This was confirmed in the results of triangulation between measures of change in the variables which indicate strong patterns of associations between changes in specific beta frequencies of frontal lobe activity, forehead temperature and self-reported feelings of core affect (arousal) and task engagement for the Right Dominant group, as shown in Table 1. Beta activity is known to replace alpha during diffuse arousal and cognitive activity and it is normally associated with an externally focused, attentive, problem solving state. The correlation results indicate that the demand conditions of the stimuli were different, eliciting changes in the underlying hemispherical activity of the EEG that were reflected in the temperature changes measured at the left and right supraorbital regions. The patterns of associations observed between changes in beta activity in the frontal EEG and temperature change were positive for the MAP and negative for the iMAP stimuli.

In response to the non-contact interaction with the MAP stimulus, the Right Dominant group exhibited a pattern of statistically significant positive correlations between the EEG and temperature measures. Positive associations existed between changes in right hemisphere temperature and beta activity (SMR \& low Beta) at the $95 \%$ level or better. 
Regression analysis identified a strong linear relationship existed between changes in right hemisphere temperature and low beta and that the gradient of the curve is statistically significant at the $95 \%$ level $(t=2.623, p=.024)$ as illustrated on the left in figure 3. Greater changes were evident in right hemisphere physiological measures. Mean temperature decreases were observed on both hemispheres however, a greater mean decrease occurred in right hemisphere temperature and a mean increase in arousal was reported. The observed temperature decrease was significantly associated with a relative reduction in right hemisphere activation and greater left hemisphere activation. Self-reports of task engagement indicated that this group considered the MAP stimulus to be more agreeable in comparison with the iMAP, and also more agreeable in comparison with the Left Dominant group.

In contrast, contact based interaction with the iMAP stimulus resulted in a pattern of statistically significant negative correlations between the EEG and temperature measures for the Right Dominant group. Negative associations were evident between changes in left hemisphere temperature and beta activity (SMR \& low Beta) at the $95 \%$ level or better. Both left and right temperature change were positively associated with arousal change and negatively with task Engagement at the $95 \%$ level or better. Regression analysis identified strong linear relationships existed between changes in left hemisphere temperature and arousal and that the gradient of the curve is statistically significant at the $99 \%$ level $(t=3.886, p=.003)$ as illustrated on the right in Figure 3. Greater changes were evident in mean left hemisphere physiological measures of temperature and the correlated beta frequencies. Mean temperature increases were observed on both hemispheres. However, a greater mean increase in left hemisphere temperature was observed and a mean decrease in arousal was reported. This group considered the iMAP stimulus to be less agreeable in comparison with the MAP, and also found it less agreeable in comparison with the Left Dominant group.

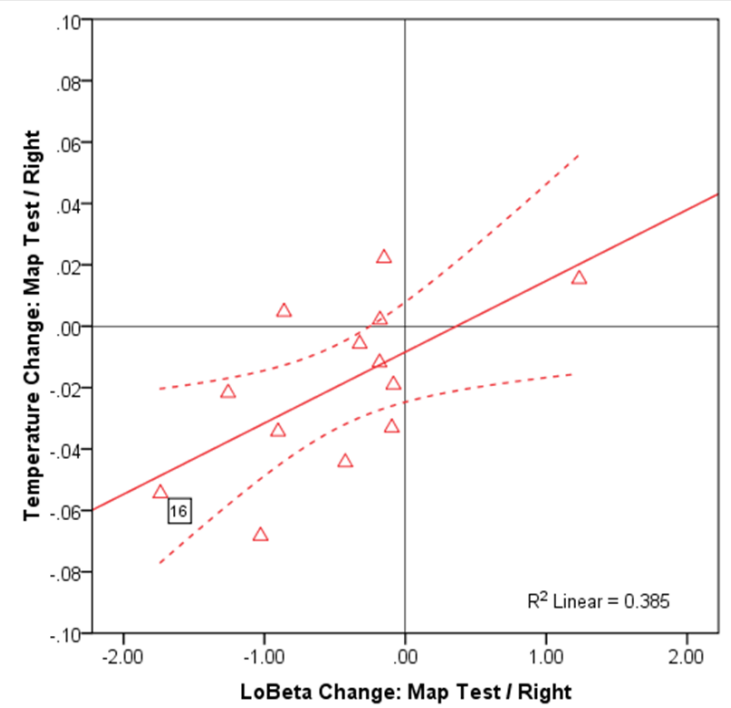

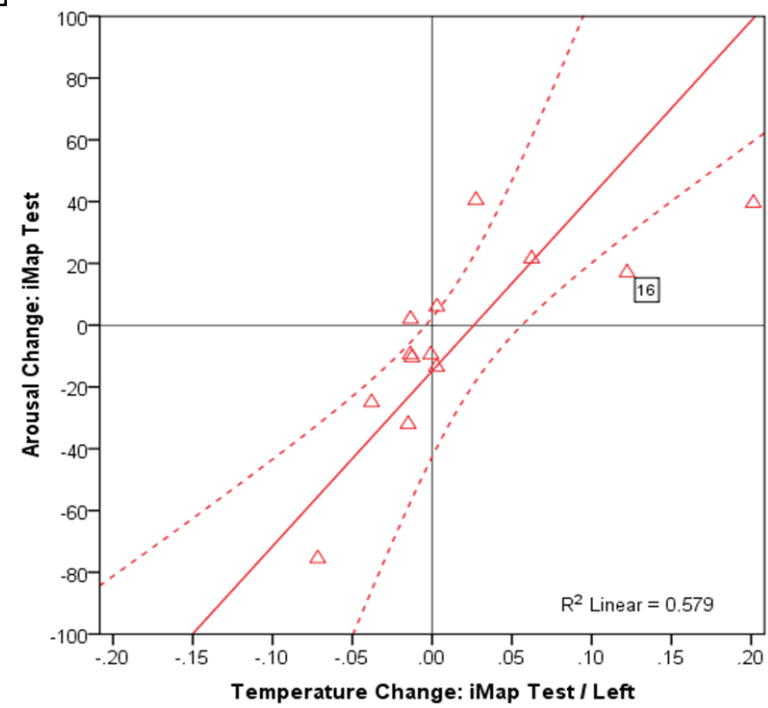

Fig 3. Scatter plots of significant correlations with strong linear relationships: RIGHT dominant sub-group

Table 1. Summary of correlations between changes in Temperature, EEG and self-reported Core Affect.

\begin{tabular}{|c|c|c|c|c|c|c|c|c|c|}
\hline & & \multicolumn{4}{|c|}{ MAP TEST } & \multicolumn{4}{|c|}{ IMAP TEST } \\
\hline & & \multicolumn{2}{|c|}{$\begin{array}{l}\text { LEFT Dominant } \\
(N=20)\end{array}$} & \multicolumn{2}{|c|}{$\begin{array}{l}\text { RIGHT Dominant } \\
(N=13)\end{array}$} & \multicolumn{2}{|c|}{$\begin{array}{l}\text { LEFT Dominant } \\
(N=20)\end{array}$} & \multicolumn{2}{|c|}{$\begin{array}{c}\text { RIGHT Dominant } \\
(N=13)\end{array}$} \\
\hline & & LH & RH & LH & RH & LH & RH & LH & RH \\
\hline \multicolumn{2}{|c|}{ Spearman's rho $(\rho)$} & \multicolumn{2}{|c|}{$\Delta \mathrm{T}$} & \multicolumn{2}{|c|}{$\Delta \mathrm{T}$} & \multicolumn{2}{|c|}{$\Delta \mathrm{T}$} & \multicolumn{2}{|c|}{$\Delta \mathrm{T}$} \\
\hline SMR $12-15 \mathrm{~Hz}$ & $\Delta s \beta$ & -.062 & -.048 & $.610^{*}$ & $.819^{\star *}$ & .251 & .377 & $-.698^{* *}$ & -.038 \\
\hline Low $\beta$ 16-20Hz & $\Delta I \beta$ & -.134 & -.174 & .374 & $.610^{*}$ & .042 & $.671^{\star *}$ & $-.593^{*}$ & .038 \\
\hline $\operatorname{High} \beta 20-23 \mathrm{~Hz}$ & $\Delta h \beta$ & -.268 & -.102 & -.368 & .418 & .329 & .245 & -.275 & -.027 \\
\hline Arousal & $\Delta \mathrm{A}_{\mathrm{r}}$ & .102 & -.319 & -.093 & .049 & .430 & .397 & $.867^{\star *}$ & $.754^{* *}$ \\
\hline Valence & $\Delta \mathrm{V}_{\mathrm{a}}$ & .102 & .337 & .352 & .527 & .232 & .159 & .126 & .060 \\
\hline Engagement & $E_{n}$ & -.126 & .163 & .000 & -.060 & .029 & -.200 & $-.632^{*}$ & $-.725^{\star *}$ \\
\hline \multicolumn{10}{|c|}{$\begin{array}{l}\text { **. Correlation coefficient is significant at the } 0.01 \text { level (2-tailed). } \\
\text { *. Correlation coefficient is significant at the } 0.05 \text { level ( } 2 \text {-tailed). } \\
\text { LH - Left Hemisphere } \\
\text { RH - Right Hemisphere }\end{array}$} \\
\hline
\end{tabular}




\section{Conclusions}

These results provide conclusive evidence in support of the proposal that two distinct 'types', defined by the differentia of forehead temperature asymmetry trait, exist within the population sample. Triangulation between the initial baseline measures of temperature asymmetry, alpha asymmetry and arousal was successful for the Right Dominant subgroup only. Furthermore, the observation that both these physiological measures have a negative association with the reported level of arousal indicate that IRT and the EEG are both measuring similar tonic states along the arousal dimension of core affect. This suggests that for this particular sub-group, the observed temperature asymmetry trait is indicative of an underlying trait disposition in cortical activity that is influential in core affective state and cognitive processing during interaction with a complex stimulus. This appears to be a key differentiating characteristic between the two groups and it may be inferred that the initial trait of these groups has been influential both in the nature of the evoked response and the effectiveness of its measurement. The observed pattern of correlations between measures of change in the variables clearly indicates that the two groups identified within the sample have responded differently to the stimuli. When analysed as a total sample $(N=33)$, the significant correlation coefficients were not apparent, indicating the occurrence of a 'masking' effect arising from the complexity of the sample and the contrasting responses, which has not been considered or reported in previous research. Furthermore, the direct contrast in the pattern of correlations observed in the results of the Right Dominant group clearly demonstrates that the nature of the level of interaction (contact or non-contact) has been influential in the changes evoked by the stimuli.

The results demonstrate that the variation in the demand condition elicited by the stimuli influenced the degree of psychophysiological arousal and this was in turn influenced by the underlying asymmetry traits in the observed subgroups. It was also noted that the reactions of individual participants within a sub-group might be varied in response to the same stimulus. These observations can be effectively explained by recognised empirical principles in the discipline of Psychophysiology that explicate individual differences in tonic and phasic arousal in terms of the extent of Autonomic Balance in an individual which is linked to the complimentary principles of the Law of Initial Values (LIV). The dominance of the Sympathetic (SNS) or Parasympathetic (PNS) nervous systems will be influential in the action of homeostatic processes and the initial level of vascular tonus (i.e. vaso-constriction or dilation). Therefore, the pre-stimulus state of the individual may be identified as an important consideration in IRT measurement as the higher the initial level of temperature then, in keeping with the LIV, either a smaller increase or a greater decrease in temperature will be observed in response to a stimulus capable of producing an increase or decrease in physiological arousal. Also, empirical research based on the concept of Individual Response Specificity has found that an individual may demonstrate greater sensitivity to changes in their own autonomic responses, which can influence both their actual levels of autonomic reactivity as well as their self-reports of these reactions [24]. As the Right Dominant group exhibited significantly lower initial left hemisphere temperature values in comparison to the Left Dominant group, it follows that increases in left hemisphere temperature will be more readily observable in this group. As both groups exhibit comparable temperatures on the right hemisphere then it follows that either increases or decreases in right hemisphere temperature will be observable in both groups.

The results of the Right Dominant group indicate that a decrease in right hemisphere temperature is a marker of a relative decrease in right hemisphere beta activity during the non-contact interaction with the MAP stimulus. The perceptible feelings of increased arousal evoked by this phasic state change have created a more preferable state of core affect and a more positive (approach or appetitive) behavioural response to the MAP stimulus for this particular group. In contrast, the increase in left hemisphere temperature is a marker of a relative decrease in left hemisphere activation during the contact interaction with the iMAP stimulus. Notably, a greater relative increase in right hemisphere Beta was observed in the raw data for this group although this was not correlated with right hemisphere temperature change. The perceptible feelings of decreased arousal evoked by this phasic change have created a less preferable state of core affect and a negative (aversive or withdrawal) behavioural response to the iMAP stimulus for this particular group. These manifestations find concordance with the reviewed literature regarding the role of hemispherical activation of the frontal lobes [27] and forehead temperature dynamics and affective states, i.e. increase|negative and decrease|positive [17].

It is known that temperature decreases of the skin are an indication of vasoconstriction and temperature increases are an indication of vasodilation in healthy control subjects. Therefore, the conclusion may be drawn that the asymmetric phasic activation of the frontal lobes observed in response to the variation in the level of interaction with the stimuli has resulted in asymmetric sympathetic activity of the forehead vascular system. This conclusion also posits that the underlying tonic asymmetry in activity of the frontal lobes will explain the initial temperature asymmetry trait observed in the experimental samples.

This is an important conclusion as the review of the recent IRT literature identified that asymmetry in forehead temperature and the possible causation of this is a line of research that has not been effectively explored to date. Some theories and empirical studies support the proposal made here that asymmetric cortical and sympathetic activity play a functional role in forehead temperature dynamics. For example, it has been proposed that cortical control and representation of autonomic function is asymmetrical and that this autonomic opponency is the foundation of feelings of core affective state $[19,20]$. Functional asymmetry of the sympathetic nervous system has also been demonstrated in studies of facial blood flow using pulse transducers $[52,53]$. In one of the few examples of medical research into facial 
temperature asymmetry, using thermistor sensors, significantly higher right blood flow and temperature in the forehead (but not in the cheeks) has been attributed to hemispheric asymmetry of sympathetic vascular control [54]. Also, a recent study of activation in the frontal lobe during a continuous performance task using a combination of fNIRS, fMRI, and peripheral physiological measurements (including skin blood flow measured using laser Doppler flowmetry) concluded that task-evoked sympathetic arterial vasoconstriction was the physiological origin of a decrease in venous volume, and that these changes in sympathetic outflow may accompany almost any cognitive and emotional process [55]. Importantly, this work identifies that the target vessels of this region of interest are innervated by nerve fibres that run along the internal carotid artery and, as it has been established that the supraorbital and supratrochlear vessels are supplied directly by the internal carotid via the frontopolar branch of the anterior cerebral artery, then a relationship between asymmetry in frontal cortical activity, sympathetic control of blood flow and forehead temperature asymmetry may be postulated with some confidence.

It is evident then that raw temperature data derived from thermal imaging can be used for effective evaluation of the cognitive and affective states evoked by interaction with complex systems, which offers great potential for research fields such as Psychology, Human-Computer Interaction $(\mathrm{HCl})$ and User Experience Design (UX). However, the research presented here demonstrates the need for continued empirical investigation of the relationship between forehead temperature dynamics and frontal cortical activity to further establish the efficacy of IRT and develop its application within these fields. It is suggested that future research is required to establish the long-term stability and reliability of the observed temperature asymmetry characteristics as a means of categorising user 'types'.

Finally, the results presented here give credence to the view that variation in the levels of interaction demanded by the stimuli influenced underlying frontal cortical processes altering sympathetic control of forehead blood flow. Furthermore, the evidence indicates that the differentia of tonic (baseline) forehead temperature asymmetry provides an index of underlying trait frontal EEG activation that moderates cognitive and affective reactivity to the stimuli presented. An important finding of this work is that when the data for the two sub-groups was analysed as one set, the results of the triangulation were unsuccessful and no significant correlations were observed. However, triangulation between variables was successful with the group exhibiting right temperature dominance, indicating that this sub-group presents more readily discernible and measureable patterns of association between cortical activity and temperature dynamics. This physiological marker may therefore be used to effectively distinguish between different 'types' of subjects within a sample population, and provides a new lens through which to view the exploration of cognitive and affective states using facial skin temperature and IRT.

\section{References}

[1] Pavlidis, I., Levine, J., \& Baukol, P., "Thermal imaging for anxiety detection". Proceedings of the 2000 IEEE Workshop on Computer Vision Beyond the Visible Spectrum: Methods and Applications, South Carolina (USA), pp. 104-9, 2000.

[2] Puri, C., Olsen, L., Pavlidis, I., Levine, J., \& Starren, J., "StressCam: Non-contact measurement of users' emotional states through thermal imaging". Proceeddings of the 2005 ACM Conference on Human Factors in Computing Systems (CHI 2005), Portland, Oregon (USA), pp. 1725-1728, 2005.

[3] Yun, C., Shastri, D., Pavlidis, I., \& Deng, Z., "O' game, can you feel my frustration?: Improving user's gaming experience via StressCam". Proceedings of the 2009 ACM Conference on Human Factors in Computing Systems (CHI 2009 - New Input Modalities), Boston, MA (USA), pp. 2195-2204, 2009.

[4] Shastri, Pavlidis, \& Wesley, 2009

[5] Levine, J. A., Pavlidis, I. T., MacBride, L., Zhu, Z., \& Tsiamyrtzis, P., "Description and clinical studies of a device for the instantaneous detection of office-place stress". Work: A Journal of Prevention, Assessment and Rehabilitation, 34 (3), 359-364, 2009.

[6] Zhu, Z., Tsiamyrtzis, P., \& Pavlidis, I., "Forehead thermal signature extraction in lie detection". Proceedings of the 29th Annual International Conference of the IEEE Engineering in Medicine and Biology Society, Lyon, (France), pp. 243-246, 2007.

[7] Zhu, Z., Tsiamyrtzis, P., \& Pavlidis, I., "The segmentation of the supraorbital vessels in thermal imagery". Proceedings of the 5th International Conference on Advanced Video and Signal Based Surveillance (AVSS 2008), Santa Fe (New Mexico), pp. 237-244, 2008.

[8] Kang, J., McGinley, J. A., McFayden, G., \& Babski-Reeves, K., "Determining learning level and effective training times using thermography". Proceedings of 25th Army Science Conference (CD Rom). Orlando, FL (USA), NTIS, 2006.

[9] Or, C. K., \& Duffy, V. G., "Development of a facial skin temperature-based methodology for non-intrusive mental workload measurement". Occupational Ergonomics, 7, 83-94, 2007.

[10] Merla, A., \& Romani, G. L., "Thermal signatures of emotional arousal: A functional infrared imaging study". Proceedings of the 29th Annual International Conference of the IEEE. IEEE Engineering in Medicine and Biology Society, Lyon (France), pp. 247-249, 2007.

[11] Nakanishi, R., \& Imai-Matsumura, K., "Facial skin temperature decreases in infants with joyful expression". Infant Behaviour \& Development, 31, 137-144, 2008. 
[12] Nozawa, A., \& Tacano, M., "Correlation analysis on alpha attenuation and nasal skin temperature". Journal of Statistical Mechanics: Theory and Experiment, (1), 1-9, 2009.

[13] Yuen, P., Hong, K., Chen, T., Tsitiridis, A., Kam, F., Jackman, J., et al., "Emotional \& physical stress detection and classification using thermal imaging technique". 3rd International Conference on Crime Detection \& Prevention (ICDP 2009), IEEE: IET Conference Publications, pp. 1-6, 2009.

[14] Reyes, Lee, Liang, Hoffman, \& Huang, 2009

[15] Nhan, B., \& Chau, T., "Classifying Affective States Using Thermal Infrared Imaging of the Human Face". Transactions on Biomedical Engineering, 57 (4), 979-987, 2010.

[16] Zajonc, R. B., "Emotion and Facial Efference: A theory reclaimed". Science, 228 (4695), 15-21, 1985.

[17] Zajonc, R. B., Murphy, S. T., \& Inglehart, M., "Feeling and facial efference: Implications of the Vascular Theory of Emotion". Psychological Review, 96 (3), 395-416, 1989.

[18] MacNeilage, P. F., Rogers, L. J., \& Vallortigara, G., "Evolutionary Origins of the Left \& Right Brain". Scientific American, July 2009, 48-55, 2009.

[19] Craig, A. D., "Forebrain emotional asymmetry: A neuroanatomical basis?" Trends in Cognitive Sciences, 9 (12), 566-571, 2005.

[20] Craig, A. D., "Interoception and Emotion". In M. Lewis, J. M. Haviland-Jones, \& L. Feldman-Barrett (Eds.), Handbook of Emotions (3rd ed., pp. 272-290). New York: The Guilford Press, 2008.

[21] Damasio, A. R., "Looking for Spinoza: Joy, sorrow and the feeling brain". London: Vintage, 2004.

[22] Prinz, J., "Gut Reactions: A Perceptual Theory of Emotion". New York: Oxford University Press, 2004.

[23] Feldman Barrett, L., "Are Emotions Natural Kinds?" Perspectives on Psychological Science, 1 (1), 28-58, 2006.

[24] Andreassi, J. L., "Psychophysiology: Human Behaviour \& Physiological Response" (5th ed.). New York: Psychology Press, Taylor \& Francis Group, 2007.

[25] Coan, J. A., \& Allen, J. J., "Frontal EEG asymmetry as a moderator and mediator of emotion". Biological Psychology, 67, 7-49, 2004.

[26] Cacioppo, J. T., Tassinary, L. G., \& Berntson, G. G. (Eds.), "Handbook of Psychophysiology" (3rd ed.). New York: Cambridge University Press, 2007.

[27] Fox, E., "Emotion Science". Basingstoke, Hampshire: Palgrave Macmillan, 2008.

[28] Pollina, D. A., Dollins, A. B., Senter, S. M., Brown, T. E., Pavlidis, I., Levine, J. A., et al., "Facial skin surface temperature changes during a 'concealed information' test". Annals of Biomedical Engineering, 34 (7), $1182-$ $1189,2006$.

[29] Stemberger, J., Allison, R. S., \& Schnell, T., "Thermal Imaging as a way to classify cognitive workload". 7th Canadian Conference on Computer and Robot Vision (CRV 2010). Ottowa, Ontario (Canada): IEEE Computer Society, pp. 231-238, 2010.

[30] Nhan, B., \& Chau, T., "Classifying Affective States Using Thermal Infrared Imaging of the Human Face". Transactions on Biomedical Engineering, 57 (4), 979-987, 2010.

[31] Genno, H., Ishikawa, K., Kanbara, O., Kikumoto, M., Fujiwara, Y., Suzuki, Y., et al., "Using facial skin temperature to objectively evaluate sensations". International Journal of Industrial Ergonomics, 19, 161-171, 1997.

[32] Nozawa, A., \& Tacano, M., "Correlation analysis on alpha attenuation and nasal skin temperature". Journal of Statistical Mechanics: Theory and Experiment , 2009 (1), 1-9, 2009.

[33] Jenkins, S. D., Brown, R. D., \& Rutterford, N., "Comparing thermographic, EEG and subjective measures of affective experience during simulated product interactions". International Journal of Design, 3 (2), 53-65, 2009.

[34] Russell, J. A., "A circumplex model of affect". Journal of Personality and Social Psychology, 39 (6), 1161-1178, 1980.

[35] Russell, J. A., "Emotion, core affect, and psychological construction". Cognition \& Emotion, 23 (7), 1259-1283, 2009.

[36] Drummond, P. D., "Facial flushing during provocation in women”. Psychophysiology, 36 (3), 325-332, 1999.

[37] Vassend, O., \& Knardahl, S., "Personality, affective response, and facial blood flow during brief cognitive tasks". International Journal of Psychophysiology, 55, 265-278, 2005.

[38] Grabowski, T. J., Damasio, H., Eichhorn, G. R., \& Tranel, D. "Effects of gender on blood flow correlates of naming concrete entities". Neurolmage, 20, 940-954, 2003.

[39] Wilson, B. A., Alderman, N., Burgess, P. W., Emslie, H., \& Evans, J. J., "Behavioural Assessment of Dysexecutive Syndrome". Thames Valley Test Company, 1996.

[40] Burgess, P. W., Alderman, N., Evans, J. J., Emslie, H., \& Wilson, B. A., "The ecological validity of tests of executive function". Journal of the International Neuropsychological Society, 4, 547-558, 1998.

[41] Alderman, N., Burgess, P. W., Knight, C., \& Henman, C., "Ecological validity of a simplified version of the multiple errands test". Journal of the International Neuropsychological Society, 9, 31-44, 2003.

[42] Greer, B., \& Mulhern, G., "Making sense of data and statistics in psychology". Hampshire: Palgrave Publishers Ltd, 2002

[43] Leighton, J. P., "Internal Validity". Retrieved March 25, 2011 from Salkind, N. J., Encyclopedia of Research Design: http://www.sage-ereference.com/researchdesign/ Article_n192.html, 2010.

[44] Ring, E. F., \& Ammer, K., "The technique of infra red imaging in medicine". Thermology International, 10 (1), 7 $14,2000$. 
[45] Ring, E. F., Ammer, K., Wiecek, B., Plassmann, P., Jones, C. D., Jung, A., et al., "Quality assurance for thermal imaging systems in medicine". Thermology International, 17 (3), 103-106, 2007.

[46] Vardasca, R., Plassmann, P., Jones, C. D., \& Ring, E. F., "Thermal monitoring of hand stress during keyboard typing". 9th International Conference on Quantitative InfraRed Thermography, Technical University of Lodz, Krakow (Poland), pp. 169-174, 2008.

[47] Pivik, R. T., Broughton, R. J., Coppola, R., Davidson, R. J., Fox, N., \& Nuwer, M. R., "Guidelines for the recording and quantitative analysis of electroencephalographic activity in research contexts". Psychophysiology, 30, 547-558, 1993.

[48] Luck, S. J., "An Introduction to the Event-Related Potential Technique". Cambridge, MA: The MIT Press, 2005.

[49] Thompson, M., \& Thompson, L., "The neurofeedback book". Wheatridge, USA: Association for Applied Psycophysiology \& Biofeedback, 2003.

[50] Pizzagalli, D. A., "Electroencephalography and High-Density Electrophysiological Source Localisation". In J. T. Cacioppo, L. G. Tassinary, \& G. G. Berntson (Eds.), Handbook of Psychophysiology (3rd ed., pp. 56-84). New York: Cambridge University Press, 2007.

[51] de Boer, A. G., van Lanschot, J. J., Stalmeier, P. F., van Sandick, J. W., Hulsher, J. B., de Haes, J. C., et al., "Is a single-item visual analogue scale as valid, reliable and responsive as multi-item scales in measuring quality of life?" Quality of Life Research, 13 (2), 311-320, 2004.

[52] Drummond, P. D., \& Mirco, N., "Staring at one side of the face increases bloodflow on that side of the face". Psychophysiology, 41, 281-287, (2004).

[53] Drummond, P. D., \& Chung, C., "Immersing the foot in painfully-cold water evokes ipsilateral extracranial vasodilation". Autonomic Neuroscience: Basic \& Clinical, 166, 89-92, 2012.

[54] Benedicic, M., Dolenc, V. V., Stefanovska, A., \& Bosnjak, R., "Left-right asymmetry of the facial microvascular control". Clinical Autonomic Research, 16, 58-60, 2006.

[55] Kirilina, E., Jelzow, A., Heine, A., Niessing, M., Wabnitz, H., Bruhl, R., et al., "The physiological origin of taskevoked systemic artefacts in functional near infrared spectroscopy". Neuroimage, 61, 70-81, 2012. 